\title{
e-Migrinter
}

$7 \mid 2011$

Réflexions croisées sur les migrations en Afrique de l'Ouest

\section{Entrer par le logement pour étudier des pratiques de migrants circulaires}

Quelques enseignements d'une approche quantitative et d'une approche qualitative à Ouagadougou (Burkina Faso)

Julie Chapon et Guillaume Le Roux

\section{(2) OpenEdition}

\section{Journals}

Édition électronique

URL : https://journals.openedition.org/e-migrinter/876

DOI : $10.4000 /$ e-migrinter.876

ISSN : 1961-9685

Éditeur

UMR 7301 - Migrinter

Édition imprimée

Date de publication : 1 septembre 2011

Pagination : 36-57

ISSN : 1961-9685

Référence électronique

Julie Chapon et Guillaume Le Roux, «Entrer par le logement pour étudier des pratiques de migrants circulaires », e-Migrinter [En ligne], 7 | 2011, mis en ligne le 20 novembre 2017, consulté le 20 mai 2021. URL : http://journals.openedition.org/e-migrinter/876; DOI : https://doi.org/10.4000/e-migrinter.876 


\section{$2^{\text {ème }}$ partie : \\ Pratiques urbaines des migrants}

\section{Entrer par le logement pour étudier des pratiques de migrants circulaires : quelques enseignements d'une approche quantitative et d'une approche qualitative à Ouagadougou (Burkina Faso)}

Julie Chapon \& Guillaume Le Roux

$\mathbf{N}$

otre envie de collaborer, à l'occasion de ce séminaire, s'est d'abord appuyée sur la connaissance d'un espace commun, Ouagadougou, à travers des travaux de recherche doctorale (Julie Chapon) et la participation à la mise en place et aux traitements des enquêtes «Mobilités spatiales en Afrique de l'Ouest " réalisées par l'Institut de Recherche pour le

Développement (Guillaume Le Roux). Malgré nos thématiques de recherche et nos approches différentes, la mutualisation de nos expériences nous a permis de prendre conscience de ce que nos travaux partagent : la position scientifique dans la manière d'aborder de façon globale les mobilités et la mobilisation de certains concepts et outils, notamment pour envisager les mobilités les plus labiles.

\begin{abstract}
Nous étudions des pratiques de migrants circulaires à Ouagadougou, en proposant d'utiliser comme entrée d'observation le logement, à partir duquel nous envisageons le déploiement des différentes pratiques de mobilité. Après avoir présenté les concepts orientant nos travaux, nous discutons la pertinence de l'entrée d'observation choisie, puis nous présentons les méthodes utilisées et quelques résultats issus de nos approches respectives.
\end{abstract}

Cadre théorique et contexte

Pour en comprendre toute loa mesure, la définition du concept de migration circulaire ou de circulation doit être mise en perspective avec d'autres concepts apparus progressivement dans les domaines de la géographie et de la démographie. La migration a été couramment définie comme un changement plus ou moins durable de lieu de résidence. Elle implique simultanément, outre un changement de lieu de résidence, un changement d'environnement social (Zelinsky, 1971). Ceci permet alors de s'affranchir des limites administratives souvent utilisées par les démographes et qui ne sont pas toujours pertinentes. L'auteur montre de plus une diversification et une intensification croissante des mobilités apparaissant dans les sociétés modernes dans leurs distances, durées, rythmes qui rendent plus difficile la définition du lieu de résidence. La résidence est classiquement définie en démographie 
comme «le lieu où les individus ont coutume d'habiter» (Henry, 1981). L'étude statistique et démographique nécessite de lier les individus à un lieu unique. Néanmoins ceci n'est pas toujours possible et ne rend pas toujours bien compte de la réalité (Courgeau, 1980).

Différents auteurs ont insisté sur le fait que, pour éclairer les questions de peuplement, l'analyse doit considérer toutes les formes de mobilités qu'elles soient définitives ou non et quelle qu'en soit l'échelle spatiale ou temporelle: «la migration ne constitue que l'infime partie visible d'un iceberg où toutes les formes de mobilité sont occultées par une définition trop étroite » (Courgeau, 1988 : 2). Ainsi, la définition de la mobilité spatiale (Bassand et Brulhardt, 1980) comme ensemble des déplacements des individus dans l'espace quels qu'en soient les durées, distances, moyens, causes et conséquences a posé les bases d'une conceptualisation élargie.

Les études fines des pratiques de mobilité en Afrique et en Amérique Latine ont permis des avancées dans la prise en compte de la complexité des relations entre individus et lieux, et de l'importance de la prise en compte de l'unité collective ou familiale dans l'analyse de ces relations (Dureau, 2002). La multiplication des notions employées pour désigner la localisation multiple des pratiques de mobilité témoignent, non pas de la nouveauté du phénomène, mais de l'importance de cette prise en compte dans l'étude des mobilités à différentes échelles spatio-temporelles : espace vécu (Chevalier, 1974), champ migratoire (Simon, 1984), système résidentiel (Lebris et al., 1987), territoire circulatoire (Tarrius, 1992), densité de résidence (Barbary, Dureau, 1993) etc. On désigne alors comme circulation, mobilité circulaire ou encore migration circulaire la pratique de mobilité soustendant la présence réitérée d'une personne dans ces différents pôles ou lieux.
Les circulations, telles que définies par Zelinsky, correspondent à un type particulier de mobilité spatiale, clairement distinguées des migrations (Zelinsky, 1971). Il les définit comme des mouvements souvent de courte durée, répétitifs ou cycliques et sans intention des individus de changer de résidence de façon permanente. Afin de s'affranchir de caractéristiques subjectives de durée et d'intention, nous considérons la circulation comme le mouvement répété mettant en relation deux ou plusieurs lieux de l'espace de vie d'un individu. Ces circulations sont alors caractérisées par leurs rythmes, durées de présence dans les lieux quelles que soient les échelles temporelles (à l'échelle de la vie de l'individu jusqu'au quotidien) et spatiales. Comme le fait remarquer Zelinsky, il est parfois judicieux de remplacer le lieu par sa fonction pour analyser certaines pratiques.

La migration circulaire est un phénomène ancien en Afrique de l'Ouest, avec la pratique de commerces à longue distance entre le Sahel et l'Afrique du Nord et le Sahel et les pays côtiers, la pratique du nomadisme, et les migrations liées aux études, à la diffusion de l'islam, etc. Le phénomène s'est amplifié sous la colonisation, les colonies sahéliennes ayant pour fonction de fournir de la main d'œuvre à celle du Golfe de Guinée. Les migrations saisonnières vers la côte étaient aussi motivées par un besoin de numéraire induit par l'apparition d'impôts et la monétarisation de l'économie. Ce système migratoire colonial s'est maintenu après les indépendances et ces circulations se sont installées au cœur de l'organisation sociale et économique des sociétés (Marchal, Quesnel, 1997). Si aujourd'hui elles se sont diversifiées, elles restent au centre des stratégies résidentielles et économiques familiales. Certains travaux ont pu les mettre en évidence par l'introduction de la notion de système de résidence comme «ensemble articulé de lieux de résidence des membres d'une famille étendue ou élargie » (Le bris $e t$ al., 1987). La migration circulaire a par 
exemple un rôle important, dans un contexte de forte croissance démographique et urbaine, en termes d'accès au logement où la pluri-localisation des familles permet de faire face à la densification des parcelles ou à la pénurie de logement en ville, et de tirer parti des différents lieux investis. Elle est aussi liée dans certaines zones rurales à la multiplication des activités, souvent selon un rythme saisonnier lié à la pratique de l'agriculture, pour faire face aux difficultés économiques et pour palier l'insécurité alimentaire, comme l'illustrent des travaux sur diverses régions rurales du Niger (Mounkaila, 2002 ; Boyer, Mounkaila, 2009).

Les migrations circulaires, méconnues des grandes enquêtes statistiques nationales (notamment des recensements nationaux qui ne les prennent pas en compte), jouent pourtant un rôle primordial dans le fonctionnement des villes (Dupont, Dureau, 1994). A Ouagadougou, les deux enquêtes «Mobilités spatiales » (2007 et 2009) mises en place par l'IRD (Institut de Recherche pour le Développement) montrent l'importance de ce phénomène qui associe très fortement les habitants de la capitale avec le reste du Burkina Faso et avec l'étranger (avec une place privilégiée de la Côte d'Ivoire) par leurs pratiques résidentielles et par leurs circulations (Boyer et al., 2008 ; Boyer, Delaunay, 2009).

Liée à cette prise en compte, dans le champ d'étude sur les mobilités, de différentes formes de migrations circulaires, ou circulations, ainsi que de l'inscription des mobilités dans un continuum à différentes échelles spatio-temporelles, la notion de logement a été utilisée en dépassant celle de résidence entendue comme "lieu où l'on a coutume d'habiter ».

\section{Entrer par le logement pour observer les migrations circulaires et les pratiques spatiales et urbaines des migrants circulaires}

En le prenant dans un sens strictement matériel comme «un local à usage d'habitation", le logement comprend la résidence sans s'y réduire, et se révèle plus pratique pour appréhender des réalités diverses et complexes des mobilités. Il est possible, à partir du logement, de situer un individu à tout moment et quelle que soit l'échelle temporelle du séjour (en prenant en compte tous les lieux avec au moins une nuitée effectuée, ou poser un seuil de durée, comme c'est le cas dans une approche quantitative où la comparabilité est souhaitée). Aussi l'unité du logement permet de saisir au moment de l'enquête l'ensemble des occupants du logement à un instant de leur trajectoire et, de façon rétrospective, les différents logements fréquentés pour reconstituer les trajectoires des individus dans toute leur complexité. Reste à définir qui sont les occupants du logement.

La notion de ménage est généralement liée à la notion de logement. Au départ, elle reliait la famille et l'habitat, puis elle a évolué vers la définition de «l'ensemble des habitants normalement dans le logement, quel que soit leur lien de parenté, incluant donc les personnes absentes de longue durée qui sont appelées à y revenir. Il s'agit du logement habité de façon permanente, c'est à dire de la résidence principale.» (Brun, Driant, Segaud, 2003). Cette définition reste ici attachée indirectement à la notion de résidence. La définition du ménage varie selon les pays, selon deux principales orientations: soit le ménage se réduit au logement et comprend toutes les personnes vivant sous un même toit; soit l'identification du ménage est fondée sur le critère de la mise en commun des ressources et des dépenses. Dans un certain nombre de pays, un logement peut contenir plusieurs ménages, identifiés selon le critère de partage des moyens et des dépenses. 
Le logement pris comme un cadre physique se révèle pratique dans le sens où il simplifie l'unité d'observation afin de l'alimenter ensuite d'éléments sociaux, économiques, historiques, etc. On observe alors les entrées et sorties du logement sans se restreindre à la notion de résidence, tout en contournant les difficultés posées par les différentes définitions du ménage.

\section{Le « logement » dans un contexte urbain africain}

Depuis plusieurs décennies, les grandes villes d'Afrique de l'Ouest connaissent une forte croissance urbaine et démographique. Ouagadougou atteint selon les résultats du recensement de 2006 près de 1,5 millions d'habitants et sa superficie a quasiment doublé depuis 1996, avec un habitat bas et très étalé, l'expression «immeubles couchés » reflétant bien cette croissance horizontale.

Ce phénomène a focalisé de nombreux travaux d'anthropologues, de géographes, de démographes, d'architectes. Certains chercheurs ont réutilisé des notions développées dans les pays industrialisés pour comprendre ce qui se jouait dans ces villes. Cependant bien souvent cette démarche se révéla inadaptée, comme en témoigne la notion de «famille». D'autres chercheurs, à l'image de l'équipe de Le Bris (1985), se sont efforcés d'affiner leurs analyses. Intéressés par des situations complexes de l'habitat faisant souvent suite à une densification de parcelles, ils ont alors montré que « la famille ne correspond pas nécessairement à l'unité de résidence. Dans de nombreux cas ces groupes familiaux éclatent en plusieurs unités de résidence dans un même quartier, parfois dans des quartiers différents ou à la campagne. » (Le Bris, 1981, p.14). C'est dans ce contexte que ces auteurs ont proposé le concept de « système résidentiel ».

Si nous avons évoqué la notion de famille et la difficulté de son approche dans un contexte urbain africain, c'est bien parce que la désignation des types d'habitation y est souvent liée. Il est ainsi délicat d'utiliser les termes de ménage, mais aussi de «concession» ou encore de «cour» qui semblent simples mais restent flous et renvoient indirectement à la notion de famille. C'est en ce sens que le logement pris comme cadre physique à usage d'habitation (avec accès direct sur l'extérieur ou sur des parties communes de la parcelle) est plus simple à manier: il prend en compte la diversité des situations, et peut être pertinent dans une étude sur des pratiques de circulations.

\section{Intérêt de cette entrée pour l'étude des circulations}

L'avantage d'une entrée par le logement est d'avoir accès à un panel de données exploitables de façon multi scalaire. D'abord par sa localisation: "position relative dans la ville du moment, la localisation du logement exprime un choix en faveur d'un certain type de rapport à l'agglomération (taille, confort, statut d'occupation etc.), la localisation détermine, selon les groupes sociaux un certain accès aux ressources offertes dans les lieux de la ville: les emplois, les équipements, les services, les commerces, etc. Elle détermine aussi l'accès aux ressources d'une autre nature, dans un espace structuré par les pratiques de ces lieux, l'expérience accumulée au cours de la vie et les relations avec les habitants de ces lieux» (Dureau, 2002, p.378). Le logement peut également être pris comme un point fixe en relation avec les lieux pratiqués par les individus dans la ville, et c'est à partir de celui-ci que vont se déployer des mobilités diverses du point de vue temporel et spatial, pour atteindre ces lieux de ressources (Boyer, 2010). Les différentes formes de mobilités dépendent étroitement de la relation entre individus et logement, on peut également analyser ces entrées et sorties du logement en terme de flux, comme un passage de frontière, qu'il est possible de mesurer à toutes les échelles spatio-temporelles. 


\section{Présentation des méthodes et des approches dans le cadre de deux thématiques de recherche différentes}

\section{Une étude quantitative des circulations et des dynamiques urbaines}

Dans l'étude du peuplement d'une ville et des dynamiques urbaines, la prise en compte des mobilités spatiales aux différentes échelles spatio-temporelles est essentielle. Depuis l'appropriation de l'approche biographique dans les études quantitatives (Haeringer, 1972), une série d'innovations méthodologiques sont apparues pour permettre la prise en compte, dans l'analyse rétrospective, des différentes échelles de mobilité ainsi que les reconstitutions de la trajectoire professionnelle, de la composition de l'unité familiale et des périodes de co-résidence (Courgeau, 1988; GRAB, 1999). Le logement, comme unité d'observation, permet alors d'avoir un point fixe autour duquel s'articule l'ensemble des formes de mobilités et de sociabilités. On peut jouer sur la «fenêtre» de temps sur laquelle on observe les mobilités individuelles afin de reconstituer le continuum des mobilités. L'approche biographique est reproduite sur la « fenêtre » de temps considérée. Dans une telle perspective, de nombreux choix méthodologiques doivent être faits face aux contraintes des réalités d'un passage d'enquêtes. Nous prendrons à titre d'exemple trois modules du questionnaire de l'enquête «Ouaga 2009 » (Boyer, Delaunay, 2009) qui illustrent l'aboutissement des méthodes biographiques sur la position scientifique qui est la nôtre ${ }^{1}$.

\footnotetext{
1 Voir aussi l'enquête «Movilidad espacial» issue d'une réflexion commune, réalisée à Bogota en 2009, source principale de mon travail de recherche doctorale s'intégrant dans le programme ANRMETAL. Le projet METAL (Métropoles d'Amérique latine dans la mondialisation: reconfigurations territoriales, mobilité spatiale, action publique, sous la direction de F. Dureau, financé dans le cadre Programme ANR/AIRD «Les Suds aujourd'hui ») porte sur Bogotá, Santiago du Chili et São Paulo. L'objectif est d'étudier les reconfigurations socio-
}

Pour assurer une représentativité spatiale de l'échantillon, le mode de sondage aréolaire est particulièrement adapté. 1965 logements ont ainsi été enquêtés à Ouagadougou en 2009 et 216 en 2007. Il peut s'effectuer sur la base d'une image satellite (Dureau, Barbary, 1991) ou à l'aide d'un Système d'Information Géographique renseigné par les techniques GPS (Boyer et al., 2008). Dans un premier temps, des parcelles ou ilots sont tirés aléatoirement. Puis après le recensement des logements de la parcelle ou de l'ilot, un ou plusieurs logements sont sélectionnés et leurs occupants sont enquêtés. Il reste encore à préciser ce que l'on désigne sous le terme de « occupant du logement». Afin de capter un large spectre d'individus mobiles, il a été décidé, pour l'enquête «Ouaga 2009» comme dans les enquêtes IRD en Amérique latine, de saisir à la fois les résidents habituels qu'ils soient présents ou non ainsi que les résidents temporaires présents ou absents à la condition qu'ils aient passé au moins trente jours (consécutifs ou non) dans le logement au cours de l'année. Cette dernière catégorie de résident peut être sousévaluée par les déclarations des enquêtés, mais cette position montre bien la volonté de saisir les individus les plus mobiles et en particulier les circulants.

Deux stratégies sont utilisées afin de saisir les mobilités quotidiennes : (1) recenser tous les déplacements de la veille avec les heures de départ, lieux d'arrivée, motifs, moyens de transport, temps de trajet, individus participant au déplacement selon une méthode biographique, (2) faire une grille des déplacements hebdomadaires selon les motifs et les lieux fréquentés (afin de garantir un meilleur recensement des divers déplacements de la semaine écoulée) avec les nombres de déplacements, fréquences, lieux de départ, moyens de transport et individus participant au déplacement.

spatiales en cours dans les métropoles d'Amérique latine depuis les années 1980, en particulier l'évolution du peuplement et les nouvelles formes de différenciation sociale à l'intérieur de l'espace urbain. 
Un module s'attache spécifiquement à saisir les pratiques résidentielles à l'échelle d'une année : la multi-résidence, la migration temporaire, saisonnière et circulaire. Comme il n'est pas possible, pour des raisons simples de mémoire des enquêtés, de collecter les informations sur une période plus longue, la «fenêtre » de temps utilisée est une année. On saisit alors tous les séjours dans les logements où l'enquêté a au moins passé une nuit et on s'attache à déterminer les dates, lieux, durées, régularités ainsi que les motifs, et types de logement dans le cas où l'individu a séjourné plus de 30 jours pendant l'année dans le logement. Les migrations circulaires sont difficiles à saisir puisque leur régularité ne se présente pas sur une seule année. Une question spécifique sur la régularité est introduite dans le questionnaire pour savoir si le séjour était répété sur les trois dernières années mais cette simple précision ne lève que peu d'ambiguités. Le questionnaire met en parallèle un calendrier des activités afin de lier la trajectoire résidentielle avec la trajectoire professionnelle et d'affiner l'analyse des systèmes de résidence en rapport avec des systèmes d'activité.

La biographie permet de saisir l'ensemble de la trajectoire résidentielle des individus à l'échelle de leur vie. Il est nécessaire de fixer un seuil en termes de durée de résidence dans les logements pour des raisons de mémoire des enquêtés et d'analyse : le seuil est fixé à un an. Comme c'est le cas dans certaines régions rurales, les migrations saisonnières sont très présentes dans les trajectoires individuelles. Cependant ces critères de collecte ne permettent pas de les saisir et un individu ayant passé toute sa vie à circuler de manière saisonnière entre deux lieux apparaitra comme quelqu'un qui n'a jamais changé de logement. Il est donc nécessaire dans certains cas de développer ce point et de l'intégrer dans la saisie des biographies. Les caractéristiques du logement et de son occupation sont précisées à l'aide de questions sur la position de l'individu dans le logement, le statut d'occupation et le type du logement. Afin d'être en mesure de prendre en compte la dépendance réciproque entre mobilité spatiale et mobilité professionnelle ainsi que les liens entre changements de résidence et constitution de la famille (GRAB, 1999), les trajectoires professionnelles et familiales sont intégrées en parallèle de la trajectoire résidentielle.

Ces différents modules permettent donc de capter les circulations à différentes échelles temporelles et l'unité d'observation étant le logement (localisé dans le quartier, la ville, le pays) les échelles spatiales peuvent aussi être balayées.

Nous présentons à titre illustratif quelques résultats sur l'analyse de données collectées dans le cadre d'enquêtes utilisant ces méthodes. Notre attention porte sur des populations particulièrement mobiles: les résidents temporaires dans les logements enquêtés (saisis transversalement) et les multi-résidents (définis ici comme les individus fréquentant au moins deux logements dans l'année pendant plus de 30 jours cumulés). On s'attache à mettre en évidence l'intérêt de l'observation des mobilités à différentes échelles spatiotemporelles en montrant qu'elle permet ainsi une meilleure compréhension des dynamiques urbaines en jeu.

\section{Eléments de résultats ${ }^{2}$}

\section{Les résidents temporaires}

Les individus déclarés temporairement dans le logement enquêté ne sont pas nécessairement des circulants dans le sens où le séjour dans le logement peut n'être qu'une étape sans idée de

\footnotetext{
2 Si la source n'est pas indiquée, les données utilisées pour ces traitements proviennent de l'enquête de 2007 à Ouagadougou. Etant donné que les analyses sont parfois faites sur de petits effectifs, les validités statistiques peuvent être remises en cause. Les résultats sont principalement présentés afin d'illustrer l'efficacité de ce type d'enquête pour l'étude des mobilités.
} 
répétition, mais certains résultats montrent une place importante du logement enquêté dans les pratiques résidentielles des résidents temporaires. Les motifs de séjour dans le logement enquêté varient fortement selon le lieu de résidence principal (figure $n^{\circ} 1$ ). Pour les résidents habituels de Ouagadougou, les motifs principaux de présence dans le logement sont variés : les visites, les motifs de travail, les motifs liés à un changement de logement et le confiage. Pour les résidents habituels du reste du pays, outre un nombre important de non-réponses, on peut noter un partage important entre motifs de travail et de visites ou vacances. Pour ce qui est des résidents habituels à l'étranger, les motifs sont en grande partie les visites ou vacances. En général, les motifs de visites ou vacances (pour lesquels il serait judicieux d'approfondir les liens qui unissent les individus dans le logement enquêté) et de travail sont les plus présents.

Figure $\mathbf{n}^{\circ} \mathbf{1}$ : Motifs de séjour dans le logement enquêté des résidents temporaires selon le lieu de résidence principal.

\begin{tabular}{|c|c|c|c|c|c|c|c|c|c|c|c|c|}
\hline \multirow[b]{2}{*}{$\begin{array}{c}\text { Lieu du domicile } \\
\text { principal }\end{array}$} & \multicolumn{9}{|c|}{ Motif du séjour à Ouagadougou } & \multirow[b]{2}{*}{ Total } & \multirow[b]{2}{*}{$\%$ col } & \multirow[b]{2}{*}{ obs } \\
\hline & $\begin{array}{c}\text { Déménagent/ } \\
\text { changement de } \\
\text { logement }\end{array}$ & $\begin{array}{c}\text { Travail/ } \\
\text { recherche de } \\
\text { travail }\end{array}$ & Union & Enfant confié & Visite/vacances & $\begin{array}{c}\text { Etudes/école } \\
\text { coranique }\end{array}$ & $\begin{array}{c}\text { Santé/soins/ } \\
\text { accouchement }\end{array}$ & Autre & Manquant & & & \\
\hline ville de Ouagadougou & 17,0 & 20,7 & 2,8 & 12,6 & 36,0 & 10,9 & 0,0 & 0,0 & 0,0 & 100,0 & 33,5 & 21 \\
\hline Burkina Faso & 0,0 & 24,8 & 6,2 & 0,0 & 19,6 & 0,0 & 7,5 & 0,0 & 41,8 & 100,0 & 34,5 & 15 \\
\hline Al'étranger & 0,0 & 2,7 & 0,0 & 0,0 & 88,3 & 0,0 & 0,0 & 9,0 & 0,0 & 100,0 & 10,0 & 9 \\
\hline Manquant & 0,0 & 0,0 & 0,0 & 0,0 & 0,0 & 0,0 & 0,0 & 0,0 & 100,0 & 100,0 & 22,0 & 12 \\
\hline Total & 5,7 & 15,8 & 3,1 & 4,2 & 27,7 & 3,6 & 2,6 & 0,9 & 36,4 & 100,0 & 100,0 & 57 \\
\hline
\end{tabular}

Sources : traitements réalisés par G. Le Roux sur les données d'enquête «Mobilités spatiales 》 de 2007 (Boyer et al., 2008).

Les pratiques résidentielles sur l'année dépendent aussi du lieu de résidence principal des résidents temporaires (figure $\left.\mathrm{n}^{\circ} 2\right)^{3}{ }^{3} \mathrm{La}$ densité de résidence dans le logement enquêté sur l'année correspond à la proportion de temps passé dans le logement enquêté sur l'année écoulée (Barbary et Dureau, 1993). De même que les motifs sont variés pour les résidents

\footnotetext{
3 Cette figure présente des «boites à moustaches». Les valeurs de la boite (en bleu) représente le premier et troisième quartile, la barre du milieu la médiane. Les moustaches (première et dernière barre aux extrémités de la boite) correspondent aux plus basses et plus hautes valeurs adjacentes. Soient $\mathrm{x}$ la variable sur laquelle on calcule les valeurs adjacentes, x(i) le ième centile des valeurs ordonnées de $x$ x[25] le premier quartile et $x[75]$ le troisième quartile, si $U$ est égal à $x[75]+3 / 2(x[75]-x[25])$, la plus haute valeur adjacente.est définie par le $\mathrm{xi}$ tel que $\mathrm{x}(\mathrm{i})<=\mathrm{U}$ et $\mathrm{x}(\mathrm{i}+1)>\mathrm{U}$. et si L est égal à $\mathrm{x}[25]-3 / 2(\mathrm{x}[75]-$ $\mathrm{x}[25])$. la plus haute valeur adjacente.est définie par le $\mathrm{xi}$ tel que $\mathrm{x}(\mathrm{i})>=\mathrm{L}$ et $\mathrm{x}(\mathrm{i}-1)<\mathrm{L}$. Les points représentent alors les valeurs qui dépassent ces valeurs de part et d'autre.
}

habituels de Ouagadougou, la présence dans le logement enquêté varie très fortement, de quelques jours pour certains à plus de 6 mois pour d'autres. Un individu peut d'ailleurs désigner le logement enquêté comme étant un logement temporaire alors qu'il y passe plus de temps sur l'année que dans sa résidence principale. Les résidents habituels du reste du pays ont en grande partie de très faible densité de résidence dans le logement enquêté (moins de 30 jours sur l'année pour la majorité). Rappelons que ces présences dans le logement sont généralement pour des motifs de visites ou vacances (ce qui est cohérent avec une faible densité de résidence) ou pour des motifs de travail. Il resterait donc à détailler ce dernier point afin de lier les pratiques résidentielles aux occupations. Cette analyse n'a pas été menée mais sera possible pour l'enquête de 2009 à Ouagadougou grâce à l'introduction du calendrier des occupations sur l'année. 
Figure $\mathrm{n}^{\circ} 2$ : Densité de résidence dans le logement enquêté selon le lieu de résidence habituel des résidents temporaires.

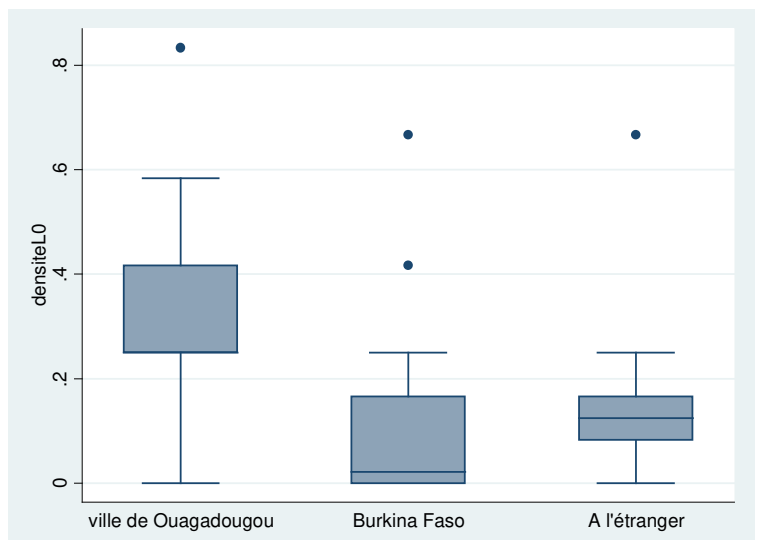

Sources: traitements réalisés par G. Le Roux sur les données d'enquête «Mobilités spatiales 》 de 2007 (Boyer et al., 2008).

Ces résidents temporaires sont pour la plupart nés hors de Ouagadougou (figure $\mathrm{n}^{\circ} 3$ ), avec une part très importante à la fois de migrants internes $(65 \%$ des résidents temporaires) et de migrants internationaux $(18 \%)$. Pourtant les localisations résidentielles des résidents temporaires sont principalement dans le centre ancien et dans la première couronne (Figure $n^{\circ} 4$ ) alors que les migrants se trouvent majoritairement en périphérie. Cette différence de localisation résidentielle témoigne de la particularité des pratiques résidentielles de ces populations temporaires ainsi que des réalités du parc de logements. Cette localisation centrale peut être liée à des stratégies individuelles d'accès aux ressources de la ville ou à la possibilité d'accueil de ces résidents temporaires. En effet, dans les quartiers centraux, plus anciens, les parcelles sont plus grandes et la capacité d'accueil y est plus importante (Boyer et Delaunay (coord.), 2009).

Figure $\mathbf{n}^{\circ} \mathbf{3}$ : Lieu de naissance des individus selon leur catégorie de résident.

\begin{tabular}{|c|c|c|c|c|c|c|c|c|}
\hline \multirow[t]{2}{*}{ Lieu de naissance } & \multicolumn{2}{|c|}{$\begin{array}{l}\text { Résident principal } \\
\text { présent }\end{array}$} & \multicolumn{2}{|c|}{$\begin{array}{l}\text { Résident principal } \\
\text { absent }\end{array}$} & \multicolumn{2}{|c|}{$\begin{array}{l}\text { Résident temporaire } \\
\text { présent }\end{array}$} & \multicolumn{2}{|l|}{ Total } \\
\hline & $\begin{array}{l}\% \\
\text { pondéré }\end{array}$ & effectif & $\%$ pondéré & $\begin{array}{l}\text { effecti } \\
\mathrm{f}\end{array}$ & \% pondéré & effectif & $\begin{array}{l}\% \\
\text { pondéré }\end{array}$ & effectif \\
\hline même secteur & 27,12 & 11 & 27,65 & 330 & 6,44 & 2 & 22,9 & 321 \\
\hline Ouagadougou & 41,18 & 6 & 17,56 & 191 & 11,23 & 9 & 17,99 & 206 \\
\hline Burkina Faso & 30,76 & 11 & 39,34 & 355 & 64,64 & 30 & 40,2 & 396 \\
\hline à l'étranger & 0,94 & 2 & 15,45 & 104 & 17,69 & 8 & 15,11 & 114 \\
\hline Total & 100 & 30 & 100 & 980 & 100 & 49 & 100 & 1059 \\
\hline
\end{tabular}

Sources : traitements réalisés par G. Le Roux sur les données d'enquête « Mobilités spatiales 》 de 2007 (Boyer et al., 2008) 
Figure $\mathbf{n}^{\circ} 4$ : Proportion de résidents temporaires présents.

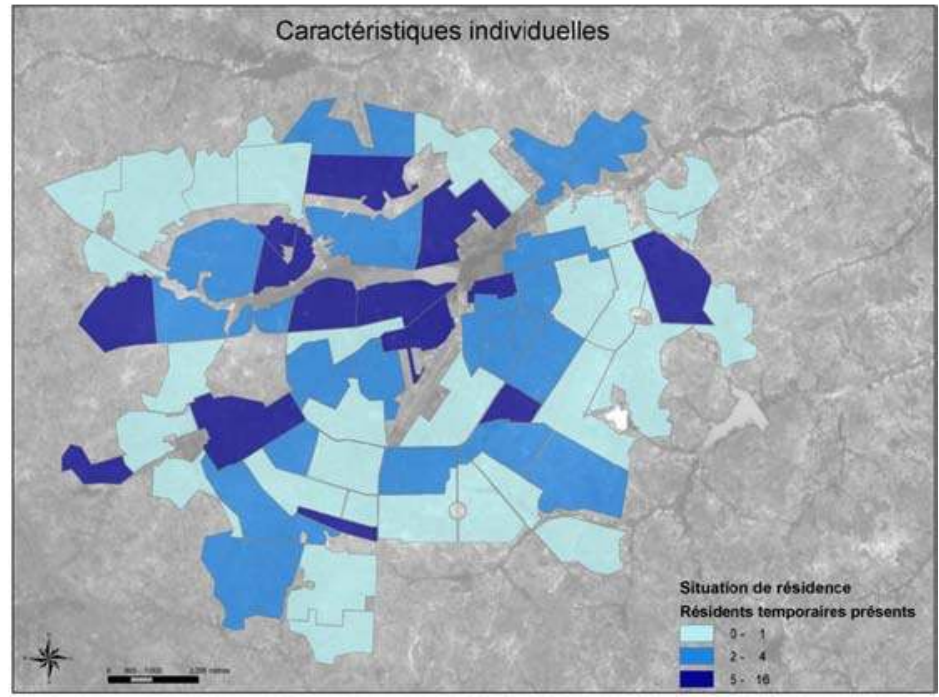

Source: Boyer et al (coord.), 2009, p. 118.

Cette localisation résidentielle particulière participe alors à des rythmes de déplacements quotidiens qui se différencient de la population résidente habituelle. Lorsque l'on analyse les grilles d'activités hebdomadaires, on remarque que le nombre de déplacements est beaucoup plus important pour les hommes en résidence temporaire (figure $n^{\circ} 5$ ). Cette différence est marquée principalement par la multiplication des activités au sein du quartier. Le choix de la localisation résidentielle semble alors important puisque le quartier de résidence est au cœur des pratiques quotidiennes des résidents temporaires.
Les rythmes se différencient aussi à l'échelle de la journée (figure $\left.n^{\circ} 6\right) .{ }^{4}$ On ne détaillera que les pratiques quotidiennes des hommes, les femmes résidentes temporaires et habituelles présentant moins de différences dans leurs pratiques quotidiennes.

\footnotetext{
${ }^{4}$ Ces graphiques horaires représentent la proportion, à chaque heure de la journée, d'individus (en bleu) présents au domicile, (en rouge) en déplacement dans le quartier de résidence, (en vert) en déplacement en dehors du quartier de résidence.
} 
Figure $\mathbf{n}^{\circ} \mathbf{5}$ : Nombre de déplacements hebdomadaires et lieux fréquentés selon la catégorie de résidence et le sexe.

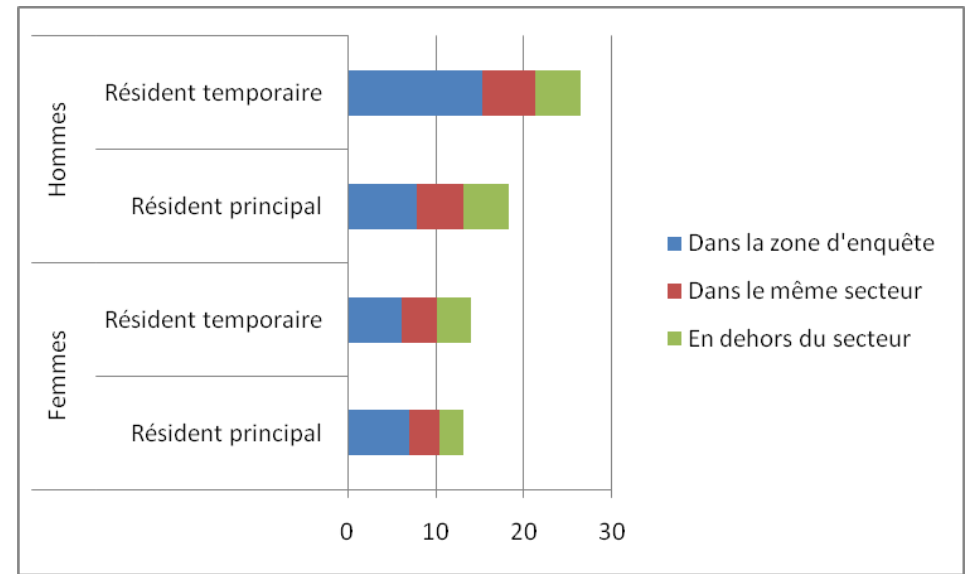

Sources : traitements réalisés par G. Le Roux sur les données d'enquête «Mobilités spatiales 》 de 2007 (Boyer et al., 2008)

Figure $\mathbf{n}^{\circ} \mathbf{6}$ : Situation des résidents temporaires et habituels selon l'heure de la journée.

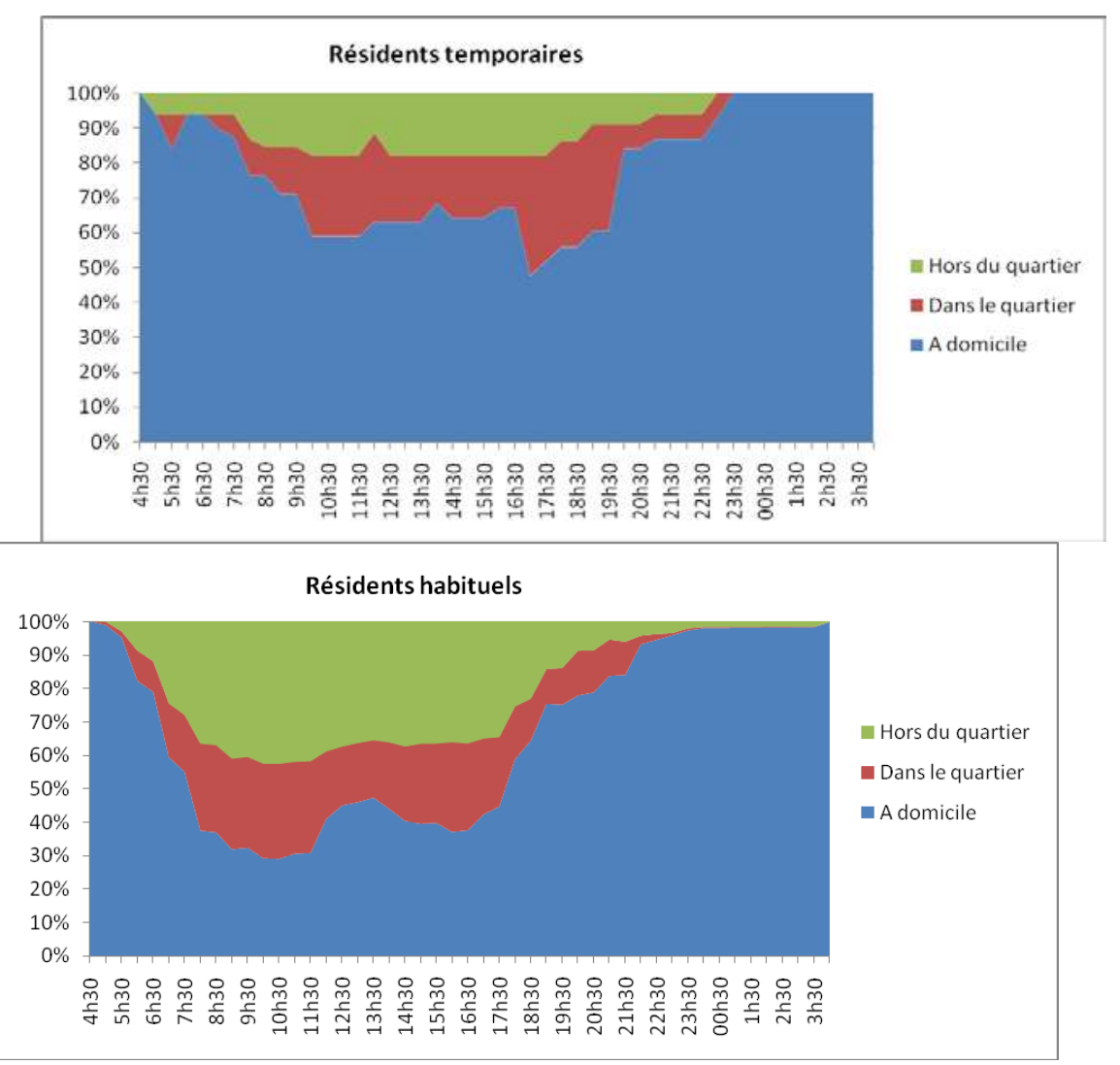

Sources : traitements réalisés par G. Le Roux sur les données d'enquête «Mobilités spatiales 》 de 2007 (Boyer et al., 2008).

Alors que les résidents habituels ont des rythmes quotidiens très liés aux horaires de tavail, les résidents temporaires ont quant à eux des rythmes plus aléatoires. Malgré un nombre de déplacements hebdomadaires plus importants, ils sont en proportion plus 
souvent dans le logement qu'en dehors. Les sorties du logement sont plus tardives et les sorties du quartier ne durent pas longtemps. Les résidents temporaires ont donc des pratiques quotidiennes plus intenses dans le quartier mais cela ne se traduit pas par une présence plus longue hors du domicile. L'articulation de la grille d'activité hebdomadaire et des déplacements journaliers permet de mettre en valeur des rythmes particuliers des résidents temporaires, centrés sur le logement.

\section{Les multi-résidents}

On définit les multi-résidents comme les individus ayant passé plus de 30 jours consécutifs ou non au cours de l'année écoulée dans un autre logement que le logement principal. Cette pratique résidentielle induit des circulations entre les différents logements occupés et près de la moitié de ces séjours ont été répétés sur les trois dernières années.

Cette pratique résidentielle est liée à l'histoire migratoire des individus : plus d'un individu sur cinq ayant effectué au moins une migration internationale au cours de sa vie est multi-résident, $12 \%$ des individus ayant effectué au moins une migration interne sont multi-résidents et seulement
$8 \%$ des individus n'ayant jamais migré en dehors de Ouagadougou sont multirésidents (figure $\mathrm{n}^{\circ} 7$ ). La mobilité résidentielle à l'échelle de la vie de l'individu est donc en lien avec les pratiques résidentielles actuelles. Les migrations contribuent certainement à l'élargissement de l'espace de vie des individus, à la dispersion de la structure familiale et ainsi à la multi-polarisation des pratiques résidentielles sur l'année.

Les pratiques quotidiennes semblent être aussi associées aux pratiques résidentielles des individus. On constate que les multi-résidents effectuent plus de déplacements hebdomadaires que les autres (Figure $n^{\circ} 8$ ). Ces différences sont accentuées pour les individus nés à Ouagadougou. Cette analyse montre des inégalités de mobilité fortes à différentes échelles entre des individus qui se déplacent très peu au quotidien et sur l'année à d'autres qui pratiquent la multi-résidence et qui se déplacent beaucoup à l'échelle du quotidien. Ces inégalités de pratiques peuvent s'expliquer soit par une différence de niveau socio-économique entre individus, soit par l'acquisition de plus grandes compétences de mobilité etc.

Figure $\mathbf{n}^{\circ} 7$ : Pratiques résidentielles selon l'histoire migratoire des individus.

\begin{tabular}{|c|c|c|c|c|c|c|}
\hline \multirow[t]{2}{*}{ Histoire migratoire } & \multicolumn{2}{|c|}{ Non-multirésident } & \multicolumn{2}{|c|}{ Multirésident } & \multicolumn{2}{|c|}{ Total } \\
\hline & \% pondéré & effectif & $\%$ pondéré & effectif & $\%$ pondéré & effectif \\
\hline N'a jamais migré & 91,89 & $\overline{88}$ & 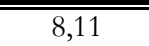 & $\overline{99}$ & $\overline{100,00}$ & 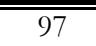 \\
\hline Migration(s) interne(s) & 87,87 & 117 & 12,13 & 23 & 100,00 & 140 \\
\hline Migration(s) internationale(s) & 78,54 & 73 & 21,46 & 20 & 100,00 & 93 \\
\hline$\overline{\text { Total }}$ & 85,72 & 278 & 14,28 & $\overline{52}$ & 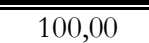 & 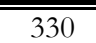 \\
\hline
\end{tabular}




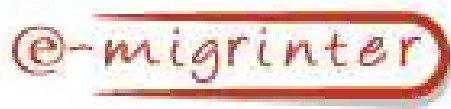

Figure $\mathbf{n}^{\circ} \mathbf{8}$ : Déplacements hebdomadaire des multi-résidents selon leur lieu de naissance.

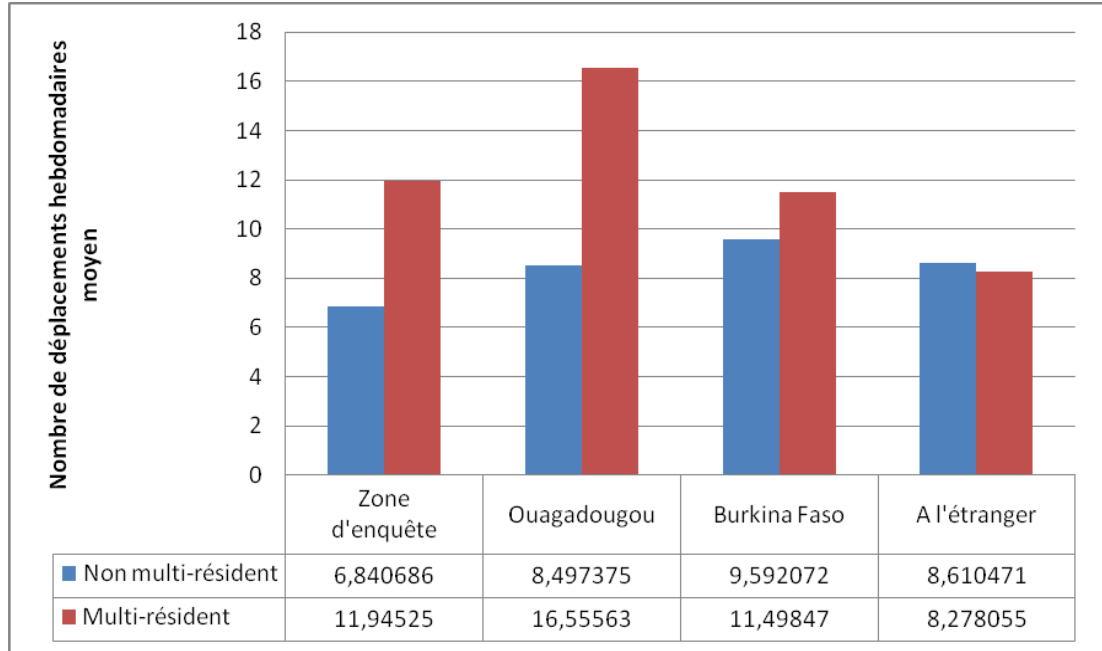

Sources : traitements réalisés par G. Le Roux sur les données d'enquête «Mobilités spatiales 》 de 2007 (Boyer et al., 2008).

Les rythmes de ces pratiques résidentielles peuvent être précisés par le calendrier résidentiel. Il peut de plus être mis en regard avec le calendrier des occupations qui permet de détailler les liens entre pratiques résidentielles et occupation. L'analyse n'a pas encore été produite pour les données de Ouagadougou mais nous montrerons à titre d'exemple celle effectuée sur le même type d'enquête dans une région rurale du Niger (Boyer et Mounkaila, 2009). Cette superposition des calendriers permet d'aider à comprendre les pratiques de migrants circulaires/saisonniers (figure $n^{\circ}$ ) et $\left.n^{\circ} 10\right)$. On peut alors observer une grande similitude entre les deux calendriers, liée aux rythmes de l'agriculture (témoignant de la multi-activité saisonnière des individus et des migrations associées) mais aussi aux rythmes scolaires (témoignant de la nécessité pour certains de migrer en ville pour l'accès à l'éducation). Dans un contexte urbain, les dynamiques ne sont évidemment pas les mêmes mais l'étude conjointe du calendrier résidentiel et des occupations permettrait de comprendre de manière plus précise certaines circulations liées à l'occupation. Les villes attirent certains de ces circulants « ruraux » et les rythmes associés contribuent alors aux dynamiques urbaines.

\section{Une approche anthropologique des pratiques de migrants circulaires nigériens à Ouagadougou}

Cette partie prendra appui sur des observations et éléments de résultats d'une approche qualitative conduite à Ouagadougou pour montrer le rôle du logement dans l'analyse de mobilités spatiales. Ce travail porte sur les pratiques de circulation de Nigériens ayant un lien avec le commerce d'artisanat (fabricants, commerçants), Ouagadougou étant l'un des lieux de leurs circulations entre différentes villes d'Afrique de l'Ouest. 
Figure $\mathbf{n}^{\circ} \mathbf{9}$ : Calendrier des occupations.

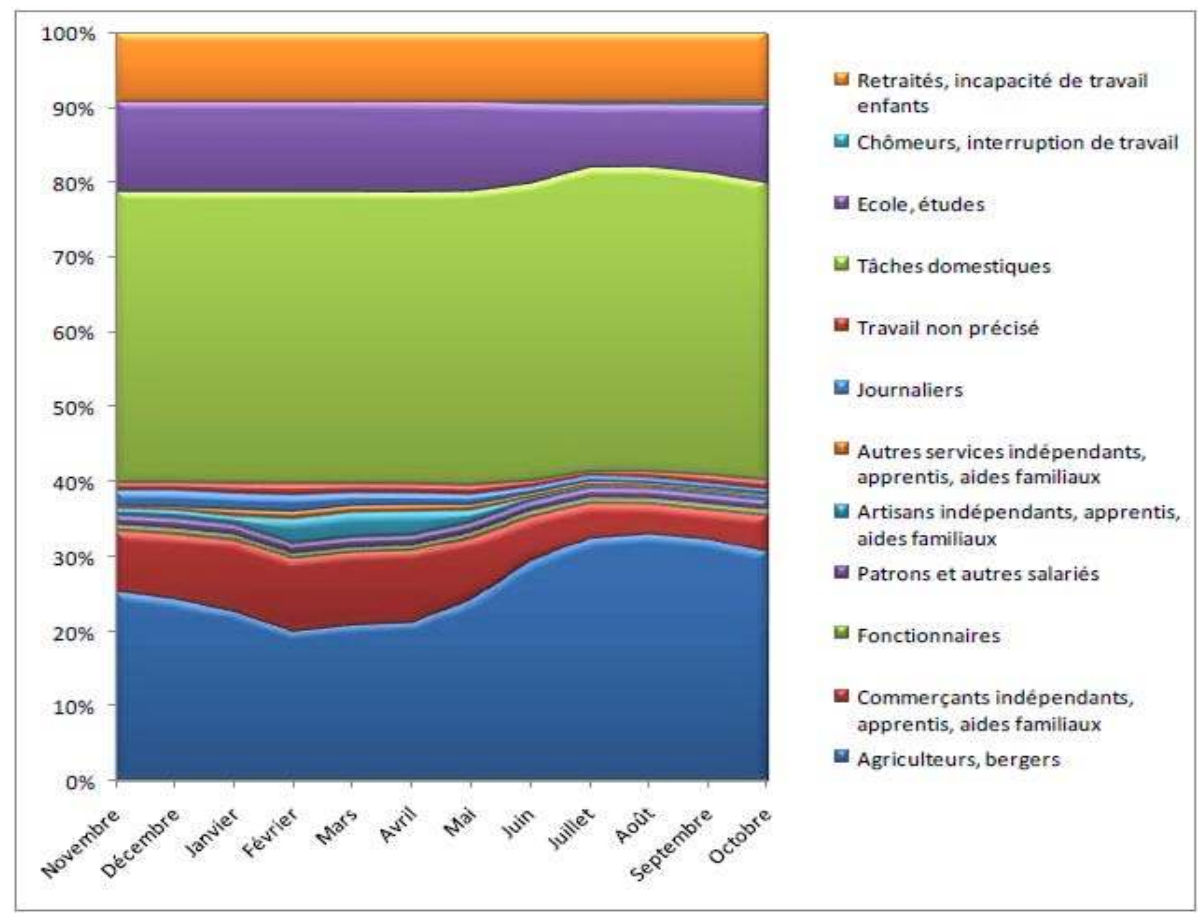

Source : Boyer, Mounkaïla, 2009, p. 98.

Figure $\mathbf{n}^{\circ} 10$ : Calendrier résidentiel.

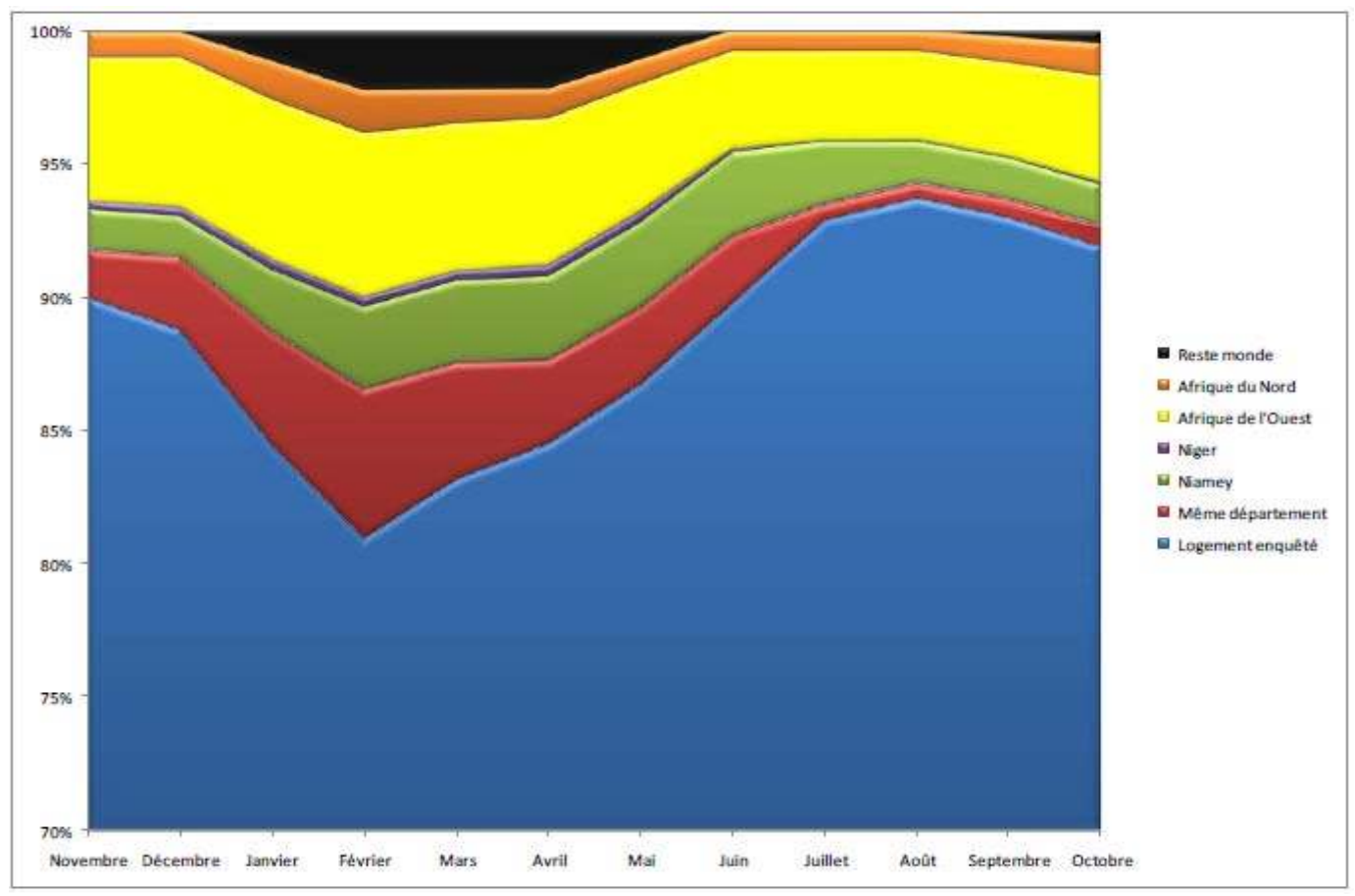

Source : Boyer, Mounkaïla, 2009, p. 98. 


\section{Précision sur l'unité « logement " à partir de données de terrains : Et la parcelle?}

Les premières enquêtes menées auprès de migrants nigériens à Ouagadougou ont montré une diversité des types de logement et des modes d'habiter en ville. L'unité d'observation du logement telle que nous l'avons présentée précédemment a été interrogée et précisée.

En effet, si le logement, pris comme cadre physique, reste utile et incontournable, sa configuration dans certaines situations n'est pas si simple à délimiter. L'exemple d'une parcelle occupée temporairement par des migrants nigériens au quartier de Dapoya au centre de Ouagadougou permet de l'illustrer (figure $\mathrm{n}^{\circ} 11$ ). L'installation des migrants se fait sur des nattes en plein air, leur présence durant l'année se situe surtout en période sèche, de décembre à juin, avec des séjours de moins d'un mois à parfois deux mois, selon les individus et les groupes, et avec une régularité variable: tous les six mois, tous les ans, tous les deux ans. Le nombre de migrants nigériens présents en même temps peut aller d'un individu à plus d'une cinquantaine.

Sur cette parcelle, d'autres migrants, des Maliens, «mendiants» en journée dans la ville, occupent également le soir un espace. Les logements en «dur» sont occupés par les membres d'une famille étendue, descendants d'un marabout premier propriétaire de la parcelle et originaire du Mali. La responsable actuelle de la parcelle est une femme veuve d'une cinquantaine d'années avec laquelle les migrants négocient leur hébergement.

Dans cette situation comment délimiter le logement des migrants nigériens? Dans l'enquête «Ouaga2009», leur occupation de l'espace ne serait pas considérée comme l'un des logements à tirer au sort, et la collecte de l'information sur leur présence dépend de la déclaration des personnes enquêtées qui peuvent omettre de les déclarer soit parce qu'ils ne dorment pas directement dans le logement, soit parce qu'ils n'ont pas de liens forts avec eux. En effet, les migrants nigériens sont hébergés sur un espace de la parcelle et non dans un logement.

Tout en gardant l'idée de la matérialisation d'un cadre physique qui sert d'habitation, le logement n'est pas forcément réduit à un local avec des murs et un toit. Ici l'installation des nattes, leur organisation, leur disposition créent physiquement une rupture sur la parcelle et peuvent permettre la délimitation d'un logement spécifique. Au cours d'une journée l'installation peut être mobile, disparaittre et se reconstruire au moment où les individus sont présents, et ainsi servir d'habitation à proprement parler au moment de son installation.

Il serait pertinent de pousser plus loin notre acception du logement pour l'entendre finalement comme le «lieu où l'on dort». Ceci nous permet ainsi de façon inductive de simplifier encore le logement tel que nous l'avions présenté.

Ainsi, pour saisir ce type de populations, il est plus pertinent de prendre en compte l'ensemble de la parcelle comme unité d'observation en considérant qu'elle est constituée d'une hétérogénéité de logements et ainsi d'analyser la complexité de son organisation. Cette démarche diffère de la précédente, dans la mesure où elle ne repose pas sur l'hypothèse que les logements d'une parcelle sont homogènes en termes d'occupation. La parcelle, comme le logement, sont à prendre en compte comme un « espace unique ».

Ainsi la particularité des méthodes d'observation qualitatives permet d'enquêter l'ensemble des individus présents quelle que soit la temporalité de cette présence, et de prendre en compte au sein de la parcelle, de par des observations prolongées et à des moments différents, ce qui se joue dans cet espace (figure $\mathrm{n}^{\circ} 11$ ). Pour exemple, ceci a 
permis de mettre en évidence des regroupements, des lieux d'échanges et des organisations identitaires de l'occupation de l'espace par les migrants selon les communautés d'origine, le lignage, les classes d'âges, etc.

L'occupation de l'espace par les migrants nigériens est assez précise : d'un côté (en rouge) les Peuls Wodaabe, les Touaregs originaires du Nord du Niger (en rose), et des lieux d'échanges durant la journée entre les deux groupes sont repérables (représentés par des points rouges), correspondant par exemple à des moments de discussion autour du thé, ou encore de partage des temps de sieste sous un hangar (photo du bas sur la figure $\mathrm{n}^{\circ} 11$ ).

L'analyse de la parcelle permet ainsi de mettre en évidence de façon fine les conditions de logement d'individus présents dans un lieu étape au sein de leurs circulations.

\section{Mobilités quotidiennes, hebdomadaires et lieux fréquentés}

Concernant les mobilités quotidiennes et hebdomadaires, il est possible, comme pour une approche quantitative de coupler différentes méthodes de collecte. Cependant, répéter les entretiens à des moments différents de la semaine, du séjour, de l'année pour un même individu, permet de réinterroger des données déjà collectées pour approfondir leur interprétation, et arriver par exemple à détailler les pratiques habituelles des individus.

Aussi par une approche qualitative, la prise en compte des lieux fréquentés par les individus peut être plus fine, à travers par exemple le suivi des individus enquêtés durant leurs déplacements, le repérage des cheminements et parcours, ainsi que les pratiques et les usages des lieux. 


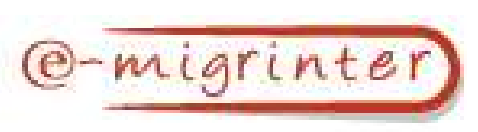

$n^{\circ} 7 \quad 2011$

Figure $\mathbf{n}^{\circ} 11$ : Parcelle dans le quartier Dapoya (Ouagadougou).
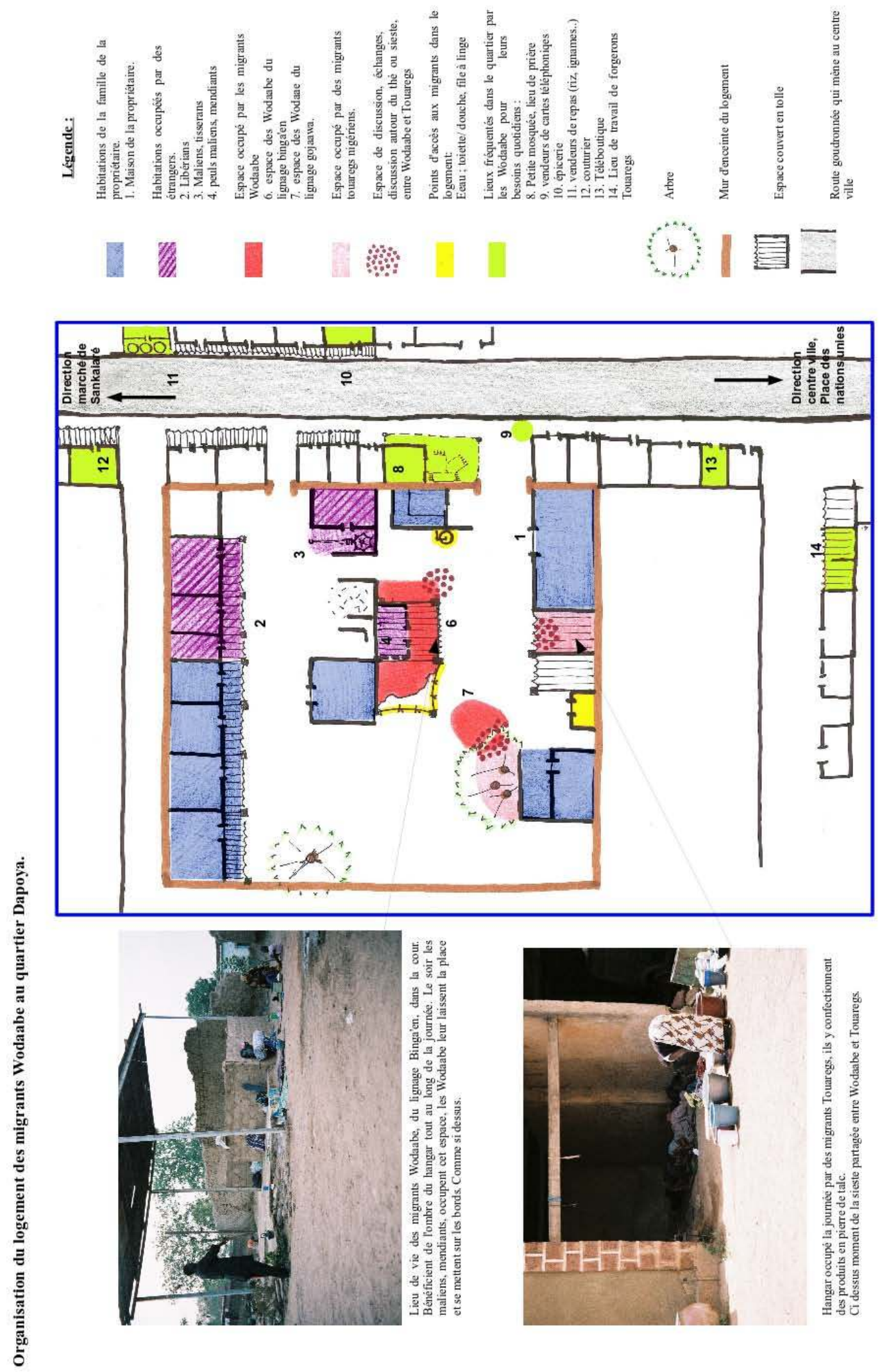

Source: J. Chapon, 2009, enquête de terrain de Master 
Cette démarche fait référence à une méthodologie, principalement développée en sociologie et en anthropologie, qui recouvre des manières diverses de collecter de l'information et qui prend en considération la position qu'entretient l'enquêteur avec le/les individus enquêtés. Il s'agit principalement de l'observation de ce qui se joue dans les lieux: il est possible ainsi d'avoir une analyse des lieux et de leurs usages par les individus, informant sur la manière dont leur position est négociée dans ces lieux. Les outils méthodologiques sont multiples que ce soit l'observation participante, les parcours commentés, la filature, etc. Les jeux de concurrence dans les lieux de vente ont pu ainsi être observés par suivi de commerçants ou par observation fixe prolongée. Les pratiques de vente ambulante des commerçants nigériens dans les rues de la ville se révèlent différentes selon la durée et la répétition des séjours ou non à Ouagadougou, etc. Certains lieux à forte concentration de commerçants locaux d'artisanat, installés dans des boutiques et/ou vendeurs ambulants, sont perçus par certains commerçants nigériens comme des lieux de tension à éviter et où des accrochages avec d'autres commerçants ont pu se dérouler. D'autres vendeurs nigériens les fréquentent en se tenant à distance, attendent que les clients terminent la tournée des boutiques pour les aborder avant leur départ. A proximité des boutiques, la position des Nigériens en tant que commerçants ambulants et étrangers est bien souvent à négocier, il n'est pas rare qu'ils soient accusés ouvertement par les commerçants locaux de « casser les prix ».

Par contre certains lieux à forte concurrence se révèlent être aussi des lieux d'alliances et d'échanges entre commerçants. En effet les abords des terrasses des restaurants et dancings de l'avenue Kwame Nkrumah sont en soirée des endroits d'affluence de vendeurs ambulants proposant une diversité de produits. Ces lieux sont généralement fréquentés parmi les commerçants Nigériens par ceux présents à Ouagadougou la majorité de l'année. À partir d'une observation prolongée sur une terrasse d'un restaurant, il a été possible de se rendre compte à la fois de moments d'accélération et de précipitation de nombreux vendeurs en même temps avec leurs produits en bout de bras, en direction des mêmes clients, suscitant parfois quelques tensions entre eux; mais aussi des moments de retrait et d'échanges sont apparus : par exemple lorsqu'un vendeur nigérien de bijoux en argent s'est écarté avec un vendeur burkinabé de bijoux en perles, a débuté une discussion, très rapidement des propositions mutuelles de produits et du troc de bijoux se sont déroulés, puis les deux individus se sont séparés, ont poursuivi leur vente ambulante dans les mêmes lieux sans forcément garder cette proximité ponctuelle. Les pratiques et les usages des lieux de vente des commerçants nigériens sont diverses et sont éclairées notamment par les pratiques et rythmes de leurs circulations.

\section{Biographie}

La seule différence concernant l'utilisation de la biographie par rapport à une approche quantitative se situe au niveau de l'élasticité du choix des échelles spatiotemporelles en prenant en compte l'ensemble des trajectoires résidentielles, professionnelles, familiale sans restriction imposée par un seuil. D'ailleurs l'exemple de migrations circulaires suivant des rythmes saisonniers (précédemment évoqué), ou plusieurs séjours répétés dans l'année, correspondent aux pratiques très variables des migrants nigériens enquêtés dans cette recherche.

Les données issues de biographies permettent également de donner des pistes d'enquêtes complémentaires. Par exemple, donner des précisions sur les différents séjours effectués par un individu à l'échelle de sa vie et faire des entretiens complémentaires pour collecter les différents 
lieux fréquentés pour chaque séjour. Ces données permettent par exemple de donner des éléments d'analyse des pratiques urbaines actuelles d'un individu, en lien avec ses pratiques au cours de séjours antérieurs dans la ville et du contexte urbain.

Les modes de vente des commerçants nigériens à Ouagadougou ont par exemple évolué face aux réglementations de la mairie vis-à-vis du droit d'installation des commerçants dans les rues. Si dans les années 90 nombreux étaient les Nigériens qui exposaient leurs produits à même le sol ou sur de petits étalages aux abords de l'école française, c'est suite à l'interdiction de ce type de pratiques par la mairie et donc au risque de se faire confisquer la marchandise, que les commerçants nigériens, présents temporairement à Ouagadougou se sont vus contraints d'effectuer de la vente ambulante ne pouvant investir dans la location d'une boutique. Les installations avec un stand se font alors au moment d'évènements culturels où un espace commercial est organisé, dans lequel ils louent un stand collectivement. Ce qui se produit dans le cadre du Fespaco (Festival panafricain du cinéma de Ouagadougou) ou encore du SIAO (Salon International d'Artisanat de l'Artisanat de Ouagadougou).

Aussi, dans les années 90 certains commerçants nigériens étaient hébergés dans la cour d'un vieux Haussa nigérien (probablement aussi commerçant) au quartier Zangouettin, dénommé l' "ancien quartier Zongo ». Un quartier populaire du centre ville qui connaissait à la fois un passage important d'étrangers et la présence d'une activité artisanale diversifiée (nombreux petits artisans de ferronniers, menuisiers, cordonniers, etc.). Suite à la mise en place d'un plan d'aménagement urbain au début des années 2000, le projet ZACA (Zone d'Activités Commerciales et Administratives) principalement centré sur de l'investissement immobilier, a concerné en partie ce quartier. Celui-ci a été détruit, les habitants ont subi un déguerpissement, et ont principalement été relogés dans d'autres quartiers en périphérie du centre ville, à la «Trame d'Accueil », à l'Est de la ville et au «Zongo » actuel à l'Ouest. Les commerçants nigériens hébergés à « l'ancien Zongo » n'ont semble-t-il pas suivi leur logeur et ont trouvé d'autres lieux d'hébergement au centre ville en mobilisant leurs réseaux de connaissances. Ils sont ainsi restés à proximité des lieux qu'ils fréquentaient, dont ceux pour la vente de leurs produits. Ceci illustre en partie les logiques résidentielles de certains commerçants nigériens, leurs pratiques actuelles ne peuvent se comprendre qu'en ayant des informations sur leurs pratiques durant leurs séjours antérieurs et sur le contexte urbain au moment de leurs séjours.

Dans cette perspective, pour une recherche portant sur des pratiques de circulation de commerçants, dans un contexte urbain d'Afrique de l'Ouest, il serait important de s'interroger sur le logement au vu des systèmes de logeurs présents dans les villes. En effet le système de « diatigui », qui correspond à un logeur qui tient également une position d'intermédiaire dans le commerce est courant dans les réseaux marchands en Afrique de l'Ouest (Schmitz, 2007 ; Chauveau, Jacob, Le Meur, 2004 ; Timera, 2000).

\section{Eléments de résultats : différentes échelles de mobilités des migrants nigériens, liées à leur activité commerciale}

Seuls deux exemples de pratiques de circulations seront exposés ici. Ces résultats sont des pistes de recherches et sont issues de données de terrains effectués en 2009 et en 2010, principalement à partir d'entretiens et d'observations.

Parmi les migrants nigériens qui effectuent des circulations liées au commerce d'artisanat, entre Ouagadougou et des villes de leur pays d'origine ou avec d'autres capitales africaines, deux groupes 
principaux à Ouagadougou se dégagent à partir de leur durée de séjour dans la ville. Ceci sera mis en perspective avec différentes échelles temporelles de mobilités liées à l'activité commerciale. D'autres situations ont été observées mais ne seront pas présentées ici.

1) (figure $n^{\circ} 12$ ) Certains migrants nigériens sont très mobiles et leur durée de séjour se résume à chaque fois à moins d'un mois et maximum 2 mois, en période sèche. Systématiquement hébergés, leur logement se situe dans un quartier ancien du centre ville, à Dapoya. Ils sont logés sur la parcelle que nous avons présentée précédemment, avec une installation sur des nattes en plein air. A la fin de leur séjour une contribution (principalement à travers une participation financière, dépendant des moyens des individus) est donnée à la propriétaire de la parcelle. Ces commerçants arrivent à Ouagadougou avec leur stock de marchandises.

Leurs mobilités quotidiennes et hebdomadaires liées à leur activité commerciale sont souvent rythmées par des sorties du logement en milieu de matinée jusqu'à midi, puis un autre moment dans l'après midi jusqu'à la tombée du jour. Les lieux fréquentés pour la vente ambulante, durant la journée, sont principalement des zones à forts potentiels de clients occidentaux résidents ou non: en semaine aux abords des écoles françaises, de restaurants, d'hôtels. Le week-end, ils sont plutôt aux abords d'hôtels équipés de piscine entre autres. Certains se rendent ponctuellement dans des boutiques d'artisanat tenues par des Occidentaux ou des Burkinabés, pour tenter de vendre en

Figure $\mathrm{n}^{\circ} 12$ : Pratiques spatiales de migrants commerçants nigériens.

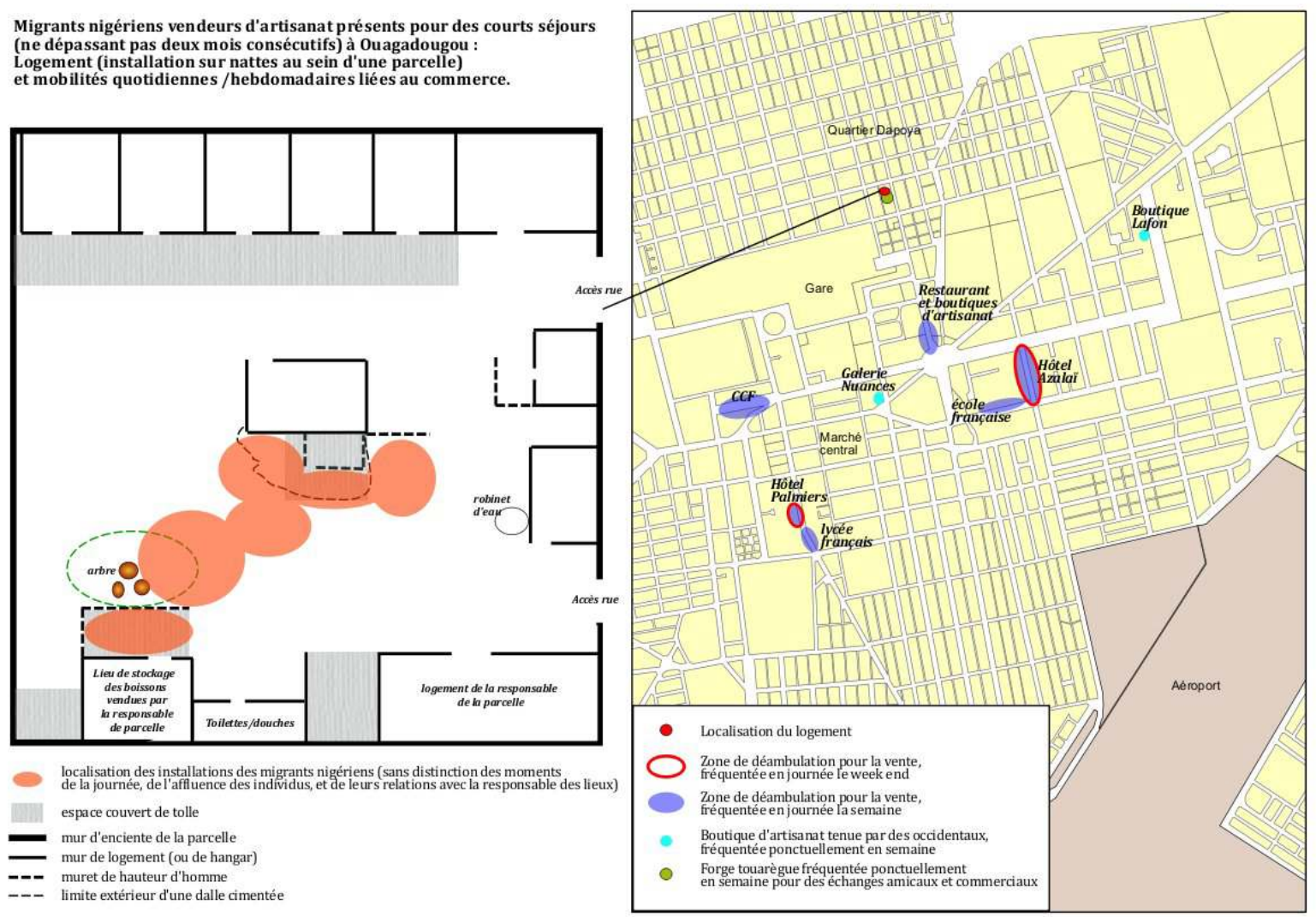

Source: J. Chapon, 2010, enquête de terrain de Thèse. 


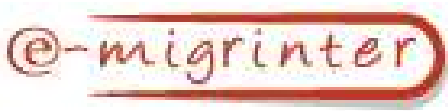

Figure $\mathbf{n}^{\circ} 13$ : Le logement de migrants nigériens travaillant dans le quartier de Dapoya.

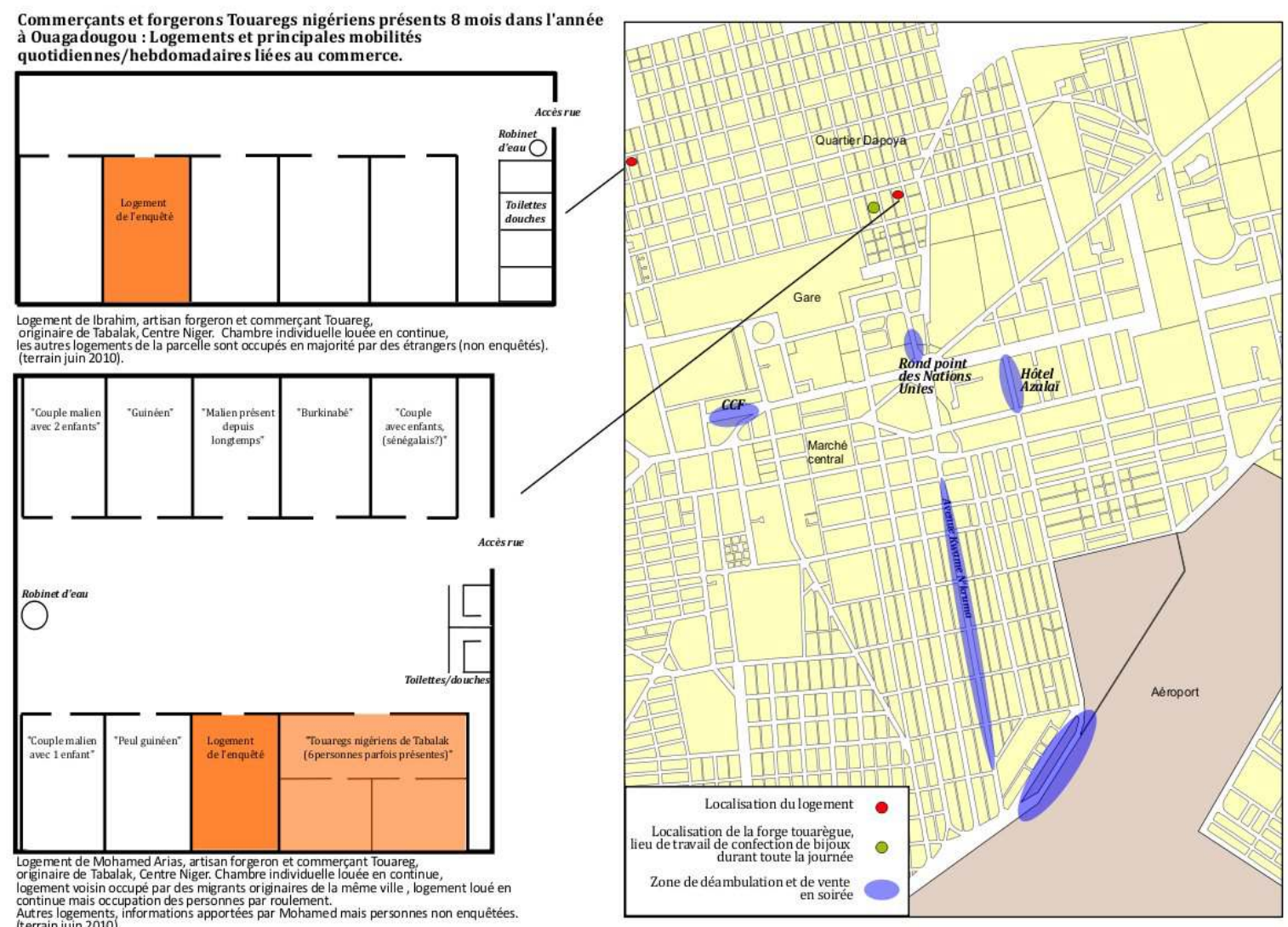

Source: J. Chapon, 2010, enquête de terrain de Thèse.

gros leurs produits. Aussi, sont réalisées régulièrement dans la semaine des visites aux autres migrants nigériens qui travaillent dans un atelier de forge pour la fabrication de bijoux, avec qui ils entretiennent des relations amicales et commerciales. Ces derniers représentent le deuxième groupe présenté.

2) (figure $n^{\circ} 13$ ) Ce sont des forgerons et commerçants Touaregs présents huit mois consécutifs dans l'année. Ils logent dans du locatif, individuel ou collectif, au centre ville, toujours au quartier Dapoya, et généralement dans des parcelles où sont aussi présents des logements occupés par d'autres migrants. Les rythmes quotidiens et hebdomadaires se réduisent en semaine, et ce durant toute la journée, au travail à l'atelier de forge. Fabricants de bijoux, ils quittent ponctuellement l'atelier pour déposer le travail effectué au client, mais souvent les commandes et transactions se font à la forge. Certains d'entre eux partent en soirée faire de la vente ambulante, dans les lieux à fort potentiel, comme l'avenue Kwame Nkrumah, le Jardin de l'amitié, etc. Ces cheminements se font selon les jours de la semaine en fonction de l'importance de la fréquentation par les usagers des lieux : pour les alentours de l'aéroport les fréquentations des vendeurs nigériens sont calquées sur les jours et horaires de départ et d'arrivée des vols et suivant les destinations. Le week-end la forge est fermée, et le temps est consacré aux visites amicales des forgerons entre eux.

Ce sont ici de premiers résultats présentés brièvement en tentant de croiser différentes formes de mobilités pour comprendre la diversité des pratiques de circulation des migrants. L'ambition de cet exposé était de montrer qu'une certaine forme de mobilité, la circulation, se précise à travers l'étude de diverses échelles de mobilités (quotidiennes, résidentielles, migrations) et que les outils méthodologiques pour les étudier 
conjointement se sont consolidés durant les Bibliographie dernières décennies. Les approches quantitatives et qualitatives exposées ici ont vocation à répondre à des problématiques différentes mais elles reposent sur des bases conceptuelles communes. Ainsi, l'étude de pratiques fines en ville d'un groupe de migrants circulaires peut se confronter à des dynamiques de peuplement à l'échelle de la ville afin de relativiser et contextualiser les pratiques observées. Inversement l'étude des pratiques de mobilité à l'échelle d'une métropole ne peut se comprendre sans des analyses plus fines des pratiques d'individus ou groupes particuliers.

Julie Chapon

doctorante MIGRINTER

UMR 6588 - CNRS/ Université de Poitiers julie chapon@hotmail.com

Guillaume Le Roux doctorant MIGRINTER

UMR 6588 - CNRS/ Université de Poitiers guillaumeleroux14@gmail.com
Barbary, Olivier ; Dureau, Françoise (1993) Des citadins en mouvement. Analyse des pratiques résidentielles à Quito (Equateur), Cabiers Sciences Humaines, vol.29, $\mathrm{n}^{\circ} 2-3$, pp. 395-418.

Bassand, Michel ; Brulhardt, Marie-Claude (1980) Mobilité spatiale. Bilan et analyse des recherches en Suisse, Lausanne, Georgi, 300 p.

Boyer, Florence; Delaunay Daniel; Le Roux, Guillaume (2008) Habiter, peupler, socialiser par les mobilités. Compte-rendu de l'Observation continue des mobilités, Rapport final, Ouagadougou, IRD, 58 p.

Boyer, Florence; Delaunay Daniel (coord.) (2009) "OUAGA. 2009 " Peuplement de Onagadongou et Développement urbain, Rapport provisoire, Ouagadougou, IRD, $250 \mathrm{p}$.

Boyer, Florence; Mounkaila, Harouna (2009) Projet de recherche:" Le Niger, espace d'émigration et de transit vers le Sud et le Nord du Sahara. Rôle et comportements des acteurs, recompositions spatiales et transformations socioéconomiques ", Rapport final, Programme FSP mobilisateur $\mathrm{n}^{\circ} 2003-74,183 \mathrm{p}$.

Boyer, Florence (2010) Mobilité, pauvretés : les villes interrogées. Croissance urbaine, statut migratoire et choix résidentiels des ouagalais. Vers une insertion urbaine ségréguée ?, Revue Tiers Monde, n²01, pp. 4764.

Brun, Jacques ; Driant Jean Claude ; Segaud Marion (dir) (2003) Dictionnaire critique de l'babitat et du logement, Paris, Armand Colin, $451 \mathrm{p}$.

Chauveau, Jean Pierre; Jacob, Jean Pierre; Le Meur, Pierre Yves (2004) L'organisation de la mobilité dans les sociétés rurales du Sud, numéro spécial: Gouverner les hommes et les ressources. Dynamiques de la frontière interne, Autrepart, n³0, pp. 3-23.

Chevalier, Jacques (1974) Espace de vie ou espace vécu ? L'ambiguité et les fondements du concept d'espace vécu, L'Espace Géographique, n¹, p. 68. 
Courgeau, Daniel (1980) Analyse quantitative des migrations humaines, Paris, Masson, 225 p. (Collection d'anthropologie physique, $\mathrm{n}^{\circ} 2$ ).

Courgeau, Daniel (1988) Méthodes de mesure de la mobilité spatiale, Paris, Éditions de l'Institut national d'études démographiques, 301 p.

Dupond, Véronique; Dureau, Françoise (1994) Rôle des mobilités circulaires dans les dynamiques urbaines. Illustrations à partir de l'Équateur et de l'Inde, Tiers-Monde, tome 35 n¹40, pp. 801-829.

Dureau, Françoise ; Barbary, Olivier (1991) L'enquête par sondage sur l'image satellite: une solution pour améliorer l'observation des populations citadines, in La qualité de l'information dans les enquêtes, Association pour la Statistique et ses Utilisations, Dunod, pp. 365-397.

Dureau, Françoise (2002) Les systèmes résidentiels : concepts et applications, in L'accès à la ville. Les mobilités spatiales en questions, Paris, L'Harmattan, pp. 355-382.

GRAB (1999) Biographies d'enquête. Bilan de 14 collectes biographiques, Paris, INED-PUF, 336 p. (Collection Méthodes et Savoirs, n³).

Haeringer, Philippe (1972) Méthodes de recherche sur les migrations africaines. Un modèle d'interview biographique et sa transcription synoptique, Cabier des Sciences Humaines, vol. IX, n² 4, pp. 439-453.

Henry Louis (1981) Dictionnaire démographique multilingue, volume français, Liège, UIESPOrdina Editions, 179 p.

Le Bris, Emile (1981) Contenu géographique et contenu social de la notion de résidence. Quelques résultats à partir d'enquêtes biographiques effectuées à Lomé (Togo) et Accra (Ghana), Cabiers d'études africaines, vol. 21 n $81-83$, pp. 129-174.

Le Bris, Emile; Marie, Alain; Osmont, Annick; Sinou Alain (1985) Résidence, stratégies, parenté dans les villes africaines, Les Annales de la recherche urbaine, $\mathrm{n}^{\circ} 25$, pp. 13-30.
Le Bris, Emile; Marie, Alain; Osmont, Annick; Sinou Alain (1987) Famille et résidence dans les villes africaines. Dakar, Bamako, SaintLouis, Lomé, Paris, L'Harmattan, 268 p. (Villes et entreprises).

Marchal, Jean-Yves ; Quesnel, André (1997) Dans les vallées du Burkina Faso, l'installation de la mobilité, in La ruralité dans les pays du Sud à la fin du XXe siècle, Paris, ORSTOM Editions, pp. 595-614.

Mounkaila, Harouna (2002) De la migration circulaire à l'abandon du territoire local dans le Zarmaganda (Niger), Revue européenne des migrations internationales, vol. $18 \mathrm{n}^{\circ} 2$, pp. 161187.

Schmitz, Jean (2007) Des migrants aux «notables» urbains: les communautés transnationales des gens du fleuve Sénégal (Sénégal/Mali/Mauritanie), in E. Boesen et L. Marfaing, Les nouveaux urbains dans l'espace Sabara-Sahel. Un cosmopolitisme par le bas, Paris, Karthala ; Berlin, ZMO, pp. 91-134.

Simon, Gildas (1984) Transferts de revenus et projets immobiliers de travailleurs migrants, Etudes méditerranéennes, nº, pp. 1136.

Tarrius, Alain (1992) Les fourmis d'Europe. Migrants riches, migrants pauvres et nouvelles villes internationales, Paris, L'Harmattan, 207 p. (Logiques sociales)

Timera, Mahamet (2000) Hospitalité et hébergement dans un réseau migratoire local et international d'Afrique de l'Ouest, in Lévy-Vroélant C. (dir.) Logements de passage. Formes, normes, expériences, Paris, l'Harmattan, pp. 51-67.

Zelinsky, Wilbur (1971) The Hypothesis of the Mobility Transition, Geographical Review, $n^{\circ}$ 61, pp. 210-249. 